\title{
ON WAVE FRONTS PROPAGATION IN MULTICOMPONENT MEDIA
}

\author{
BY
}

\author{
M. I. FREIDLIN
}

\begin{abstract}
The behavior as $t \rightarrow \infty$ of solutions of some parabolic systems of differential equations of the Kolmogorov-Petrovskii-Piskunov type is investigated. The present approach uses the Kac-Feynman formula and estimates on large deviations.
\end{abstract}

The behavior as $t \rightarrow \infty$ of solutions of some parabolic systems of differential equations of the Kolmogorov-Petrovskii-Piskunov (KPP) type [1] is treated in this paper. Our approach is based on the following two ideas: First, the solution of the system under consideration satisfies some integral equation in the space of functions with integration over the space of trajectories of an appropriate Markov process. Secondly, we will make use of limit theorems for the probabilities of large derivations. A similar approach has been used in [2]. We shall formulate accurate assertions and provide proof in the simplest nontrivial situation, and then indicate possible generalizations.

So, let us consider the Cauchy problem

$$
\begin{array}{ll}
\frac{\partial u}{\partial t}=\frac{D_{1}}{2} \frac{\partial^{2} u}{\partial x^{2}}+c_{11}(u, v) u+c_{12} v, & u(0, x)=g_{1}(x), \\
\frac{\partial v}{\partial t}=\frac{D_{2}}{2} \frac{\partial^{2} v}{\partial x^{2}}+c_{21} u+c_{22}(u, v) v, & v(0, x)=g_{2}(x) .
\end{array}
$$

We make the following hypotheses:

1. $D_{1}, D_{2}, c_{12}, c_{21}$ are positive constants.

2. The functions $c_{11}(u, v)$ and $c_{22}(u, v)$ are bounded from above and Lipschitz continuous.

3. The vector field $\left(c_{11}(u, v) u+c_{12} v, c_{21} u+c_{22}(u, v) v\right)$ on the plane $(u, v)$ has an unstable equilibrium at the point $(0,0)$ and a stable equilibrium at the point $(a, b), a>0, b>0$. All integral curves starting at the points of the set $\{(u, v)$ : $\left.u \geqslant 0, v \geqslant 0, u^{2}+v^{2} \neq 0\right\}$ are attracted to the point $(a, b)$ as $t \rightarrow \infty$.

4. $c_{11}(0,0)=\max _{u \geqslant 0, v \geqslant 0} c_{11}(u, v), c_{22}(0,0)=\max _{u \geqslant 0, v \geqslant 0} c_{22}(u, v)$.

5. One can choose $\alpha, h \geqslant 0$ and a function ${ }^{1} H(u, v)$ such that the following conditions hold:

Received by the editors December 21, 1981.

1980 Mathematics Subject Classification. Primary 35B40; Secondary 35K22, 60J60.

Key words and phrases. Nonlinear diffusion, wave fronts, parabolic systems.

${ }^{1} H(u, v)$ is Lyapunov's function of the stable equilibrium point $(a, b)$. The specific character of hypothesis 5 is that this function is assumed to be defined in a large domain and to be convex downwards. 
(a) $H(a, b)=0, H(u, v)>0$ for $(u, v) \in A_{\alpha / 2}=\{(u, v): u \geqslant 0, v \geqslant 0 ;(u, v) \neq$ $(a, b) ; u+v>\alpha / 2\}$.

(b) The derivative $d H / d t$ of the function $H(u, v)$, by virtue of the system

$$
\frac{d u}{d t}=c_{11}(u, v) u+c_{12} v, \quad \frac{d v}{d t}=c_{21} u+c_{22}(u, v) v
$$

satisfies the inequality

$$
\frac{d H(u, v)}{d t} \leqslant-\rho\left((u-a)^{2}+(v-b)^{2}\right),
$$

where $\rho(z), z \geqslant 0$, is an increasing continuous function, $\rho(0)=0$.

(c) The function $H(u, v)$ is convex downwards.

(d) $c_{11}(u, v)+c_{21}>0, c_{22}(u, v)+c_{12}>0$ for $(u, v) \in B_{\alpha}=\{(u, v): u \geqslant 0, v \geqslant$ $0, u+v \leqslant \alpha\}$.

Let $\nu(t), t \geqslant 0$, be a Markov process with two states, 1 and 2, for which

$$
P\{\nu(t+\Delta)=j \mid \nu(t)=i\}=c_{i j} \Delta+0(\Delta), \quad \Delta \rightarrow 0, i \neq j .
$$

Consider the process $X_{t}$ defined by the equality

$$
d X_{t}=\sqrt{D_{\nu(t)}} d W_{t}, \quad t>0, X_{0}=x \in R^{1},
$$

where $W_{t}$ is a Wiener process, $D_{1}, D_{2}$ are the coefficients of system (1). It is easily seen that the couple $\left(X_{t}, \nu_{t}\right)$ forms a Markov process in the state space $R^{1} \times\{1 ; 2\}$.

The following theorem is based on an idea belonging, apparently, to M. Kac. This is the idea of using the representation of some linear parabolic system via the process $\left(X_{t}, \nu_{t}\right)$.

TheOREM 1. Suppose that hypotheses 1 and 2 hold. Then the solution $(u(t, x)$, $v(t, x))$ of problem (1) obeys the following equations:

$$
\begin{aligned}
& u(t, x)=E_{x, 1} g_{v(t)}\left(X_{t}\right) \exp \left\{\int_{0}^{t} c\left(\nu_{s}, u\left(t-s, X_{s}\right), v\left(t-s, X_{s}\right)\right) d s\right\}, \\
& v(t, x)=E_{x, 2} g_{\nu(t)}\left(X_{t}\right) \exp \left\{\int_{0}^{t} c\left(\nu_{s}, u\left(t-s, X_{s}\right), v\left(t-s, X_{s}\right)\right) d s\right\},
\end{aligned}
$$

where $c(1, u, v)=c_{11}(u, v)+c_{12}, c(2, u, v)=c_{22}(u, v)+c_{21}$. System (2) has a unique solution bounded in every band $\left\{x \in R^{1}, 0 \leqslant t \leqslant T\right\}, T<\infty$.

Proof. If $(u(t, x), v(t, x))$ is a solution of problem (1), then equations (1) can be looked upon as linear equations, where $c_{u}(u(t, x), v(t, x))=\tilde{c}(t, x, i)$ are known functions of $t$ and $x, i=1,2$.

For the functions, smooth enough in the first argument, the infinitesimal generator $A$ of the process $\left(X_{t}, \nu_{t}\right)$ has the form

$$
A f(x, i)=\frac{D i}{2} \frac{\partial^{2} f}{\partial x^{2}}-c_{i j} f(x, i)+c_{i j} f(x, j) ; \quad i, j \in\{1,2\}, i \neq j, x \in R^{1} .
$$

So equalities (2) turn into a version of the Kac-Feynman formula for the equation

$$
\frac{\partial w(t, x, i)}{\partial t}=A w+\tilde{c}(t, x, i) w .
$$


The second assertion of Theorem 1 is straightforward from the fact that the mapping

$$
\begin{gathered}
\left(u^{0}(t, x), v^{0}(t, x)\right) \rightarrow\left(u^{1}(t, x), v^{1}(t, x)\right), \\
u_{1}(t, x)=E_{x, 1} g_{v(t)}\left(X_{t}\right) \exp \left\{\int_{0}^{t} c\left(v_{s}, u^{0}\left(t-s, X_{s}\right), v^{0}\left(t-s, X_{s}\right)\right) d s\right\}, \\
v_{1}(t, x)=E_{x, 2} g_{v(t)}\left(X_{t}\right) \exp \left\{\int_{0}^{t} c\left(v_{s}, u^{0}\left(t-s, X_{s}\right), v^{0}\left(t-s, X_{s}\right)\right) d s\right\}
\end{gathered}
$$

is a contractive one in the space $C_{0, t_{0}}$ (in the uniform norm) for sufficiently small $t_{0}>0$ (cf. [3]). Here the constant $t_{0}$ depends only on the Lipschitz constant of the functions $c_{i i}(u, v), i=1,2$, and on $\sup _{u, v, i} c_{i i}(u, v), \sup _{x_{i}}\left|g_{i}(x)\right|$.

For the sake of simplicity, let $g_{1}(x)=a \chi_{x<0}(x), g_{2}(x)=b \chi_{x<0}(x)$, where $\chi_{x<0}(x)$ is the indicator function of the set $\{x<0\} \subset R^{1}$. Just as in the case of the KPP equation, one can expect that, for large $t$, the solution $(u(t, x), v(t, x))$ of system (1) has the form of a wave propagating from left to right with some velocity $c^{*}$ :

$$
(u(t, x), v(t, x)) \approx\left(U\left(x-c^{*} t\right), V\left(x-c^{*} t\right)\right) .
$$

This wave is determined by its profiles $U(\xi), V(\xi)$ and by the propagation velocity $c^{*}$. Given $c^{*}$, the profiles may be obtained as a solution of the system of ordinary differential equations, which is derived from system (1) by setting $u(t, x)=$ $U\left(x-c^{*} t\right), v(t, x)=V\left(x-c^{*} t\right)$ :

$$
\begin{array}{ll}
\frac{D_{1}}{2} U^{\prime \prime}+c^{*} U^{1}+c_{11}(U, V) U+c_{12} V=0, & \lim _{\xi \rightarrow-\infty} U(\xi)=a, \lim _{\xi \rightarrow-\infty} V(\xi)=b ; \\
\frac{D_{2}}{2} V^{\prime \prime}+c^{*} V^{1}+c_{21} U+c_{22}(U, V) V=0, & \lim _{\xi \rightarrow \infty} U(\xi)=\lim _{\xi \rightarrow \infty} V(\xi)=0 .
\end{array}
$$

A weaker version of this assertion is as follows: there is a $c^{*}$ such that

$$
\begin{aligned}
& \lim _{t \rightarrow \infty} u\left(t,\left(c^{*}+h\right) t\right)=0, \quad \lim _{t \rightarrow \infty} v\left(t,\left(c^{*}+h\right) t\right)=0 ; \\
& \lim _{t \rightarrow \infty} u\left(t,\left(c^{*}-h\right) t\right)=a, \quad \lim _{t \rightarrow \infty} v\left(t,\left(c^{*}-h\right) t\right)=b
\end{aligned}
$$

uniformly in $h \geqslant h_{0}$ for every $h_{0}>0$. It is this assertion we shall be concerned with. We shall evaluate $c^{*}$ and prove relations (3).

Let us consider the following matrix whose elements depend on a parameter $\alpha \in R^{1}$ :

$$
\left(\begin{array}{cc}
-c_{12}+\alpha D_{1} & c_{12} \\
c_{21} & -c_{21}+\alpha D_{2}
\end{array}\right) .
$$

Let $\lambda(\alpha)$ be the largest eigenvalue of this matrix. It is not hard to verify that the eigenvalues of this matrix are real. Moreover, it is readily checked that $\lambda(\alpha)$ is a nondecreasing, downward convex function. In addition, $\lambda(\alpha)$ has an asymptote with the slope $\min \left(D_{1}, D_{2}\right)$ as $\alpha \rightarrow+\infty$, and an asymptote with the slope $\max \left(D_{1}, D_{2}\right)$ as $\alpha \rightarrow-\infty$. 
We will denote by $l(\beta), \beta \in R^{1}$, the Legendre transform of the function $\lambda(\alpha)$ :

$$
l(\beta)=\sup _{\alpha}(\alpha \beta-\lambda(\alpha)) .
$$

Let, for definiteness, $D_{1}<D_{2}$. From the above listed properties of the function $\lambda(\alpha)$ it follows that $l(\beta)=\infty$ outside, $\left[D_{1}, D_{2}\right]$. On the interval $\left[D_{1}, D_{2}\right]$, the function $l(\beta)$ is finite, positive and convex downwards. It is differentiable on $\left(D_{1}, D_{2}\right)$, and its derivative tends to $+\infty$ as $\beta \rightarrow D_{2}$, and tends to $-\infty$ as $\beta \rightarrow D_{1}$.

- THEOREM 2. Suppose hypotheses 1-5 and the condition $D_{1}<D_{2}$ to be valid. Then relations (3) hold for

$$
c^{*}=\frac{\left|\lambda\left(\alpha^{*}\right)+B\right|}{\sqrt{2\left(\alpha^{*}-A\right)}},
$$

where

$$
A=\frac{c_{22}+c_{21}-c_{11}-c_{12}}{D_{2}-D_{1}}, \quad B=\frac{D_{2}\left(c_{11}+c_{12}\right)-D_{1}\left(c_{22}+c_{21}\right)}{D_{2}-D_{1}},
$$

and $\alpha^{*}$ is a root of the equation

$$
\lambda\left(\alpha^{*}\right)+B=2 \lambda^{\prime}\left(\alpha^{*}\right)\left(\alpha^{*}-A\right), \quad \lambda^{\prime}(\alpha)=\frac{d \lambda(\alpha)}{d \alpha} .
$$

Proof. First, we shall prove that for every $h>0$

$$
\lim _{t \rightarrow \infty} u\left(t,\left(c^{*}+h\right) t\right)=\lim _{t \rightarrow \infty} v\left(t,\left(c^{*}+h\right) t\right)=0 .
$$

We shall find bounds for the function $u(t, x)$; that the second limit is equal to zero can be demonstrated in a similar way. Hypothesis 4 leads to

$$
u(t, x) \leqslant E_{x, 1} g_{\nu(t)}\left(X_{t}\right) \exp \left\{\int_{0}^{t} c\left(\nu_{s}\right) d s\right\}
$$

where $c\left(\nu_{s}\right)=c\left(\nu_{s}, 0,0\right)$. Next, we note that $X_{t}=\tilde{W}\left(\int_{0}^{t} D_{\nu(s)} d s\right)$, where $\tilde{W}_{t}$ is a proper Wiener process independent of $\nu_{t}$. Relying on this, we get from (5) for $x=c t$, $c>0$,

$$
\begin{aligned}
u(t, x) & \leqslant E_{c t, 1} g_{\nu(t)}\left(\tilde{W}\left(\int_{0}^{t} D_{\nu(s)} d s\right)\right) \exp \left\{\int_{0}^{t} c\left(\nu_{s}\right) d s\right\} \\
& \leqslant \max (a, b) \cdot \operatorname{EP}\left\{\tilde{W}\left(\xi_{t}\right)>c t \mid \xi_{t}\right\} \exp \left\{A \xi_{t}+B t\right\} .
\end{aligned}
$$

Here we designate $\xi_{t}=\int_{0}^{t} D_{\nu(s)} d s$; the constants $A$ and $B$ being defined by conditions $c(i)=A D_{i}+B$ for $i=1,2$. Since

$$
P\left\{\tilde{W}\left(\xi_{t}\right)>c t \mid \xi_{t}\right\} \leqslant \text { const } \cdot \exp \left\{-\frac{c^{2} t^{2}}{2 \xi_{t}}\right\},
$$

from (6) one can infer that

$$
u(t, c t) \leqslant C_{1} E \exp \left\{-\frac{c^{2} t^{2}}{2 \xi_{t}}+A \xi_{t}+B t\right\},
$$


where $c_{1}$ is some positive constant. Let us show that, for $c>c^{*}$, the right-hand side of (7) tends to zero as $t$ grows. To this end, we shall compute the logarithmic asymptotics of the expectation as $t \rightarrow \infty$. These asymptotics are determined by the large deviations of the random process $\xi_{t} / t$ from its limit as $t \rightarrow \infty$. According to [4] (see also [5]), the deviations of order 1 as $t \rightarrow \infty$ for $\xi_{t} / t$ are described by the action function $l(\beta)$ defined by equality (4):

$$
\lim _{\delta \rightarrow 0} \lim _{t \rightarrow \infty} t^{-1} \ln P\left\{\left|\frac{1}{t} \xi_{t}-\beta\right|<\delta\right\}=-l(\beta) .
$$

From this it follows that the logarithmic asymptotics of the expectation on the right-hand side of bound (7) are as follows:

(8) $\lim _{t \rightarrow \infty} t^{-1} \ln E \exp \left\{-t\left(\frac{c^{2}}{2 \zeta_{t} / t}-A \frac{1}{t} \zeta_{t}-B\right)\right\}=-\min _{x}\left(\frac{c^{2}}{2 x}-A x-B+l(x)\right)$.

Since the function $l(x)$ is $+\infty$ outside $\left[D_{1}, D_{2}\right]$, this minimum may be taken only over $\left[D_{1}, D_{2}\right]$. The above properties of $l^{\prime}(x)$ imply that the minimum is attained inside the interval $\left(D_{1}, D_{2}\right)$. Let us show the right-hand side of equality (8) vanishes for $c=c^{*}$. We will denote by $\tilde{\alpha}=\tilde{\alpha}(x)$ the solution of the equation $\lambda^{\prime}(\tilde{\alpha})=x$. Such $\tilde{\alpha}(x)$ is defined in a unique way for $x \in\left(D_{1}, D_{2}\right)$. Then $l(x)=x \tilde{\alpha}(x)-\lambda(\tilde{\alpha}(x))$, $x \in\left(D_{1}, D_{2}\right)$. It is not hard to check that $l^{\prime}(x)=\tilde{\alpha}(x)$. The minimum of the function $f(x)=(2 x)^{-1}\left(c^{*}\right)^{2}-A x-B+l(x)$ is attained at some point $\bar{x} \in$ $\left(D_{1}, D_{2}\right)$. We will denote $\bar{\alpha}=\tilde{\alpha}(\bar{x})$. Then remembering the definition of $\tilde{\alpha}$, from the condition $f^{\prime}(x)=0$, we derive the equations

$$
\frac{\left(\lambda\left(\alpha^{*}\right)+B\right)^{2}}{4\left(\alpha^{*}-A\right) \bar{x}^{2}}+A-\bar{\alpha}=0, \quad \lambda^{\prime}(\bar{\alpha})=\bar{x} .
$$

The equation for $\alpha^{*}$

$$
\frac{\lambda\left(\alpha^{*}\right)+B}{2\left(\alpha^{*}-A\right)}=\lambda^{\prime}\left(\alpha^{*}\right)
$$

should be joined to (9). From (9) and (10) follows that

$$
\lambda^{\prime 2}(\bar{\alpha})(\bar{\alpha}-A)=\lambda^{\prime 2}\left(\alpha^{*}\right)\left(\alpha^{*}-A\right)
$$

Since $\lambda^{\prime 2}(\alpha)(\alpha-A)$ is an increasing function of $\alpha$, the last relation yields

$$
\bar{\alpha}=\alpha^{*}, \quad \bar{x}=\lambda^{\prime}\left(\alpha^{*}\right)=\frac{\lambda\left(\alpha^{*}\right)+B}{2\left(\alpha^{*}-A\right)} .
$$

Noting these equalities we arrive at

$$
\min f(x)=f(\bar{x})=\frac{\left(\lambda\left(\alpha^{*}\right)+B\right)^{2}}{4\left(\alpha^{*}-A\right) \bar{x}}-A \bar{x}-B+\bar{x} \alpha^{*}-\lambda\left(\alpha^{*}\right)=0 .
$$

So the right-hand side of (8) vanishes for $c=c^{*}$. It is easily seen that the function

$$
\gamma(c)=\min _{x}\left(\frac{c^{2}}{2 x}-A x-B+l(x)\right)
$$


increases with $c$ for $c>0$. Therefore, $\gamma(c)>0$ for $c>c^{*}$, and according to (7) and (8), $u(t, c t)<c_{1} e^{-t \gamma(c)}$ for sufficiently large $t$. The second of relations ( $\left.3^{\prime}\right)$ can be verified in a similar way.

We proceed to prove $\left(3^{\prime \prime}\right)$. The following assertion results from $\left(3^{\prime}\right)$ : For any $\delta>0$, if $h$ is small enough and $t$ is sufficiently large, then the inequality

$$
c\left(\nu(s), u\left(t-s, X_{s}\right), v\left(t-s, X_{s}\right)\right)>c\left(\nu_{s}, 0,0\right)-\frac{\delta}{2}, \quad 0 \leqslant s \leqslant t,
$$

is valid, with probability close to 1 , for the trajectories $X_{s}$ starting from the point $X_{0}=\left(c^{*}+h\right) t$. On account of (8), this implies that, for sufficiently small $h>0$ and sufficiently large $t$,

$$
\begin{aligned}
& u\left(t,\left(c^{*}+h\right) t\right)>E_{\left(c^{*}+h\right) t, 1} g_{\nu(t)} \exp \left\{\int_{0}^{t}\left(c\left(\nu_{s}\right)-\frac{\delta}{2}\right) d s\right\}>e^{-\delta t}, \\
& v\left(t,\left(c^{*}+h\right) t\right)>E_{\left(c^{*}+h\right) t, 2} g_{\nu(t)} \exp \left\{\int_{0}^{t}\left(c\left(\nu_{s}\right)-\frac{\delta}{2}\right) d s\right\}>e^{-\delta t} .
\end{aligned}
$$

First of all, we will show that, for any $h>0$ and $T$ sufficiently large,

$$
(u(t, x), v(t, x)) \in A_{\alpha / 2}=\{u>0, v>0, u+v>\alpha / 2\}
$$

for $t>T, x<(c-h) t$. Let us denote $D_{\beta}=\{(t, x):-\infty<t<\infty, 0<x<$ $\left.\left(c^{*}+\beta\right) t, u(t, x)+v(t, x)<3 \alpha / 4\right\}, \tau=\inf \left\{s:\left(t-s, X_{s}\right) \in D_{\beta}\right\}$. Below, it will be convenient for us to designate $u_{1}(t, x)=u(t, x), u_{2}(t, x)=v(t, x)$. Since $\left(X_{s}, \nu(s)\right)$ is a strong Markov process, and $\tau$ is a Markov time, relations (2) yield the equality

$$
u_{i}(t, x)=E_{x, i} u_{\nu(\tau)}\left(t-\tau, X_{\tau}\right) \exp \left\{\int_{0}^{\tau} c\left(\nu_{s}, u_{1}\left(t-s, X_{s}\right), u_{2}\left(t-s, X_{s}\right)\right) d s\right\}
$$

Hypothesis $5(\mathrm{~d})$ implies that there is $\kappa>0$ such that $c\left(i, u_{1}(t, x), u_{2}(t, x)\right)>\kappa>0$ everywhere in the domain $D_{\beta}$. Suppose that $x>\left(c^{*}-h\right) t, h>0$. Then, the time required for the "heat" process $Z_{s}=\left(t-s, X_{s}\right)$ to reach the set $\Gamma=\{(s, x)$ : $\left.s=\max \left(0, x /\left(c^{*}+\beta\right)\right)\right\}$, starting from the point $(t, x)$, tends to infinity together with $t$. By virtue of bounds (12), everywhere on the set $\Gamma$

$$
u_{i}(t, x) \geqslant K \exp \{-\delta t\}, \quad i=1,2,
$$

for some $K>0$. Choosing $\beta$ involved in the definition of the set $D_{\beta}$ sufficiently small, one can ensure that $\delta<\kappa / 2$. From these bounds, with the help of (14), we conclude that (13) holds for some $T>0$.

Now we will show that, no matter what the $h_{1}>0$,

$$
\left(u\left(t,\left(c^{*}-h_{1}\right) t\right), v\left(t,\left(c^{*}-h_{1}\right) t\right)\right) \rightarrow(a, b) \text { as } t \rightarrow \infty .
$$

Let us choose $h \in\left(0, h_{1}\right)$ and $T=T(h)$ so that (13) should hold. We put

$$
\begin{aligned}
\tilde{D}_{\delta, T}=\left\{(s, x): s>T, 0<x<\left(c^{*}-h\right) s,\right. \\
\left.\rho\left((u(s, x)-a)^{2}+(v(s, x)-b)^{2}\right)>\delta\right\},
\end{aligned}
$$


where $\delta$ is a small positive number. By (13), Lyapunov's function $H(u(s, x), v(s, x))$ is defined at the point $(u(s, x), v(s, x))$ for $(s, x) \in \tilde{D}_{\delta, T}$. We note that, for small $\Delta$, the transformation ${ }^{2}$

$$
\left(u_{1}^{0}, u_{2}^{0}\right) \rightarrow\left(u_{1}^{\Delta}, u_{2}^{\Delta}\right), \quad u_{i}^{\Delta}=\tilde{E}_{i} u_{\nu(\Delta)}^{0} \exp \left\{\int_{0}^{\Delta} c\left(\nu_{s}, u_{1}^{0}, u_{2}^{0}\right) d s\right\}
$$

is (up to an infinitesimal of a higher order) the translation along the trajectories of the dynamical system

$$
\frac{d u_{1}}{d t}=c_{11}\left(u_{1}, u_{2}\right) u_{1}+c_{12} u_{2}, \quad \frac{d u_{2}}{d t}=c_{21} u_{1}+c_{22}\left(u_{1}, u_{2}\right) u_{2} .
$$

Let $\mu$ and $\Delta=\Delta(\mu)$ be positive numbers whose choice will be specified below. We will designate by $\tau_{1}$ the first exit time of the process $\left(t-s, X_{s}\right)$ from $\tilde{D}_{\delta, T}$ : $\tau_{1}=\inf \left\{s:\left(t-s, X_{s}\right) \notin \tilde{D}_{\delta, T}\right\}$. Let us introduce into consideration the Markov time $\tilde{\Delta}=\min \left(\Delta, \tau_{1}\right)$. From the strong Markov property of the process $\left(X_{t}, v_{t}\right)$ we get the following equality:

$$
u_{i}(t, x)=E_{x, i} u_{\nu(\tilde{\Delta})}\left(t-\tilde{\Delta}, X_{\tilde{\Delta}}\right) \exp \left\{\int_{0}^{\tilde{\Delta}} c\left(\nu_{s}, u_{1}\left(t-s, X_{s}\right), u_{2}\left(t-s, X_{s}\right)\right) d s\right\}
$$

$i=1,2 ; t>\Delta$. Since the function $H\left(u_{1}, u_{2}\right)$ is convex, for $t>T+\mu, x<\left(c^{*}-h\right) t$ $-\mu$, relation (17) yields

$$
\begin{aligned}
H\left(u_{1}(t, x), u_{2}(t, x)\right)= & H\left(E_{x} \tilde{E}_{1} u_{\nu(\tilde{\Delta})} e^{\int_{0}^{\tilde{\Delta}} c d s}, E_{x} \tilde{E}_{2} u_{\nu(\tilde{\Delta})} e^{\int_{0}^{\tilde{\Delta}} c d s}\right) \\
\leqslant & E_{x} H\left(\tilde{E}_{1} u_{\nu(\tilde{\Delta})} e^{\int_{0}^{\tilde{\Delta}} c d s}, \tilde{E}_{2} u_{\nu(\tilde{\Delta})} e^{\int_{0}^{\tilde{\Delta}} c d s}\right) \\
\leqslant & E_{x} \chi_{\tilde{\Delta}=\Delta} H\left(\tilde{E}_{1} u_{\nu(\Delta)} e^{\int_{0}^{\Delta} c d s}, \tilde{E}_{2} u_{\nu(\Delta)} e^{\int_{0}^{\Delta} c d s}\right) \\
& +E_{x} \chi_{\tilde{\Delta}=\tau_{1}} H\left(\tilde{E}_{1} u_{\nu\left(\tau_{1}\right)} e^{\int_{0}^{\tau_{1}} c d s}, \tilde{E}_{2} u_{\nu\left(\tau_{1}\right)} e^{\int_{0}^{\tau_{1}} c d s}\right),
\end{aligned}
$$

where $\chi_{\tilde{\Delta}=\Delta}$ and $\chi_{\tilde{\Delta}=\tau_{1}}$ are the indicator functions of the sets $\{\tilde{\Delta}(\omega)=\Delta\}$ and $\left\{\tilde{\Delta}(\omega)=\tau_{1}\right\}$ respectively. The above inequality implies that for fixed $\mu$ and small $\Delta$,

$$
H\left(u_{1}(t, x), u_{2}(t, x)\right) \leqslant E_{x} \chi_{\tilde{\Delta}=\Delta} H\left(\tilde{E}_{1} u_{\nu(\Delta)} e^{\int_{0}^{\Delta} c d s}, \tilde{E}_{2} u_{\nu(\Delta)} e^{\int_{0}^{\Delta} c d s}\right)+o(\Delta) .
$$

Next, noting that the functions $c(i, u, v)$ and $u_{i}(t, x)$ are uniformly continuous for $t>T, x \in R^{1}, i=1,2$, inequality (18) can be rewritten in the form

$$
\begin{aligned}
& H\left(u_{1}(t, x), u_{2}(t, x)\right) \\
& \leqslant E_{x} \chi_{\tilde{\Delta}=\Delta} H\left(\tilde{E}_{1} u_{\nu(\Delta)}\left(t-\Delta, X_{\Delta}\right) e^{\int_{0}^{\Delta} c\left(\nu_{s}, u_{1}\left(t-\Delta, X_{\Delta}\right), u_{2}\left(t-\Delta, X_{\Delta}\right)\right) d s},\right. \\
& \left.\tilde{E}_{2} u_{\nu(\Delta)}\left(t-\Delta, X_{\Delta}\right) e^{\int_{0}^{\Delta} c\left(\nu_{s}, u_{1}\left(t-\Delta, X_{\Delta}\right), u_{2}\left(t-\Delta, X_{\Delta}\right)\right) d s}\right)+o(\Delta) .
\end{aligned}
$$

\footnotetext{
${ }^{2}$ By $\tilde{E}_{i}$ we denote expectation supposing that $\nu(0)=i ; i=1,2$.
} 
As it was noted before, the argument of the function $H(\cdot, \cdot)$ on the right-hand side of inequality (19) is (up to $o(\Delta))$ the translation of the point $\left(u_{1}\left(t-\Delta, X_{\Delta}\right)\right.$, $\left.u_{2}\left(t-\Delta, X_{\Delta}\right)\right)$ in the time $\Delta$ along the trajectories of system (16). Hence, by hypothesis 5(b) one can find a constant $q_{1}>0$ such that

$$
\begin{gathered}
H\left(\tilde{E}_{1} u_{\nu(\Delta)}\left(t-\Delta, X_{\Delta}\right) e^{\int_{0}^{\Delta} c\left(\nu_{s}, u_{1}\left(t-\Delta, X_{\Delta}\right), u_{2}\left(t-\Delta, X_{\Delta}\right)\right) d s}\right. \\
\left.\tilde{E}_{2} u_{\nu(\Delta)}\left(t-\Delta, X_{\Delta}\right) e^{\int_{0}^{\Delta} c\left(\nu_{s}, u_{1}\left(t-\Delta, X_{\Delta}\right), u_{2}\left(t-\Delta, X_{\Delta}\right)\right) d s}\right) \\
\leqslant H\left(u_{1}\left(t-\Delta, X_{\Delta}\right), u_{2}\left(t-\Delta, X_{\Delta}\right)\right)-q_{1} \Delta .
\end{gathered}
$$

From (19) and (20) results that there is $\Delta>0$ so small that

$$
\sup _{t>T+\mu, x<\left(c^{*}-h\right) t-\mu} H\left(u_{1}(t, x), u_{2}(t, x)\right) \leqslant \sup _{(t, x) \in \tilde{D}_{\delta, T}} H\left(u_{1}(t, x), u_{2}(t, x)\right)-q_{1} \Delta .
$$

For any $\theta$, by selecting sufficiently small $\delta$ and using this bound a sufficiently large number of times, we obtain that $H\left(u_{1}(t, x), u_{2}(t, x)\right)<\theta$ for the points $(t, x) \in \tilde{D}_{\delta, T}$ which are far enough from the boundary of the domain $\tilde{D}_{\delta, T}$. Whence, remembering that $(a, b)$ is the only point where Lyapunov's function $H\left(u_{1}, u_{2}\right)$ vanishes, we deduce (15). Our reasoning implies also that the convergence to the limit in (15) is uniform in $h>h_{0}>0$. This completes the proof in Theorem 2 .

REMARK 1. When proving relations $\left(3^{\prime}\right)$, we made use of the fact that $c(i)=A D+$ $B, i=1,2$ (see inequality (6)). If $D_{1}=D_{2}$, then such $A$ and $B$ may not exist. The argument used above cannot also be exploited for a system with more than two unknown functions. In these cases one can act as follows (for brevity, we restrict ourselves to the case of two equations): We will denote by $\lambda\left(\alpha^{1}, \alpha^{2}\right)\left(\alpha^{1}, \alpha^{2} \in R^{1}\right)$, the maximum eigenvalue of the matrix

$$
\left(\begin{array}{cc}
-c_{12}+\alpha^{1} D_{1}+\alpha^{2} c(1) & c_{12} \\
c_{21} & -c_{21}+\alpha^{1} D_{2}+\alpha^{2} c(2)
\end{array}\right)
$$

It is established that the function $\lambda\left(\alpha^{1}, \alpha^{2}\right)$ is downward convex. Let $l\left(\beta_{1}, \beta_{2}\right)$ be the Legendre transform of $\lambda\left(\alpha^{1}, \alpha^{2}\right)$. Then the velocity $c^{*}$ of the wave front propagation is the root of the equation

$$
\min _{x, y}\left(\frac{\left(c^{*}\right)^{2}}{2 x}-y+l(x, y)\right)=0
$$

It should be borne in mind that usually the function $l\left(\beta_{1}, \beta_{2}\right)$ is finite only in some interval in the plane $\left(\beta_{1}, \beta_{2}\right)$. For example, let $D_{1}=D_{2}=D, c=c(1,0,0)=$ $c(2,0,0)$. Then a simple calculation shows that $\lambda\left(\alpha^{1}, \alpha^{2}\right)=\alpha^{1} D+\alpha^{2} c, l(D, c)=0$ and $l\left(\beta_{1}, \beta_{2}\right)=+\infty$ for $\left(\beta_{1}, \beta_{2}\right) \neq(D, c)$. In this case equation (21) yields $c^{*}$ $=\sqrt{2 D c}$.

The proof of equation (21) is analogous to the reasoning in proving Theorem 2, if one notes that $l\left(\beta_{1}, \beta_{2}\right)$ is the action function describing the large deviations for the two-dimensional process $\left(\int_{0}^{t} D_{\nu(s)} d s, \int_{0}^{t} c\left(\nu_{s}\right) d s\right)($ see $[4,5])$. 
REMARK 2. If, as the initial functions, one picks any nonnegative functions whose support is bounded from above, then the wave front propagates with the velocity indicated in Theorem 2. In the case when the initial functions are positive, the wave front may propagate with a large velocity. For example, let $g_{1}(x), g_{2}(x)>0$ and $g_{i}(x) \sim \exp (-\mu x), \mu>0$, as $x \rightarrow \infty$. Then a simple modification of the argument exploited in proving Theorem 2 shows that relations (3) hold for $c^{*}$, defined by the equation

$$
\min _{x, y}\left(\mu y+\frac{(c-y)^{2}}{2 x}+l(x)-A x-B\right)=0 .
$$

The function $l(x)$ is specified by equality (4).

REMARK 3. Wave front propagation in the process described by equations (1) is the result of interaction of the two factors: first, diffusion, and secondly, multiplication and mutual conversion of particles. For the random process describing particle motion in space, one need not take diffusion. For instance, particle motion may be described by a homogeneous process with independent increments. In doing so, generators (integro-differential) of the corresponding processes with independent increments are involved in equations (1), rather than the operators $D_{i}\left(\partial^{2} / \partial x^{2}\right)$. Under certain hypotheses on regularity of these processes, it is not difficult to write down an equation for $c^{*}$. Particle motion along the $x$-axis need not be described by a Markov process necessarily. For example, let the motion $X_{t}^{i}$ of the particle of $i$ th kind be described by the differential equation

$$
\dot{X}_{t}^{i}=b\left(Y_{t}^{i}\right)
$$

where $Y_{t}^{i}$ is a diffusion process on $[-1,1]$ governed by the operator $\left(D_{i} / 2\right)\left(d^{2} / d y^{2}\right)$ for $y \in(-1,1)$ and subject to reflection at the endpoints of the interval. We observe that the couple $\left(X_{t}^{i}, Y_{t}^{i}\right)$ forms a Markov process. Then, for the concentrations $u(t, x)$ and $v(t, x)$ of the particles of the first and second kind, we have the equations

$$
\begin{gathered}
\frac{\partial u(t, x, y)}{\partial t}=\frac{D_{1}}{2} \frac{\partial^{2} u}{\partial y^{2}}+b^{1}(y) \frac{\partial u}{\partial x}+c_{11}(u, v) u+c_{12} v, \\
\frac{\partial v(t, x, y)}{\partial t}=\frac{D_{2}}{2} \frac{\partial^{2} v}{\partial y^{2}}+b^{2}(y) \frac{\partial v}{\partial x}+c_{21} u+c_{22}(u, v) v, \\
\quad x \in(-\infty, \infty), y \in(-1,1), t>0, \\
u(0, x, y)=g_{1}(x), \quad v(0, x, y)=g_{2}(x), \\
\left.\frac{\partial u(t, x, y)}{\partial y}\right|_{y= \pm 1}=\left.\frac{\partial v(t, x, y)}{\partial y}\right|_{y= \pm 1}=0 .
\end{gathered}
$$

We will assume hypotheses $1-5$ to be fulfilled, and let $g_{1}(x), g_{2}(x)>g_{0}>0$ for $x<0$ and $g_{1}(x)=g_{2}(x)=0$ for $x>x_{0}>0$. Moreover, for brevity, we will assume that $\int_{-1}^{1} b^{i}(y) d y=0, i=1,2$. Then it is possible to establish that a wave propagates 
along the $x$-axis with some velocity $c^{*}$. To determine this velocity, let us consider the eigenvalue problem ( $\alpha, \beta$ are real parameters)

$$
\begin{array}{r}
\frac{D_{1}}{2} \varphi_{y y}^{\prime \prime}+\left[\beta\left(c_{11}(0,0)+c_{12}\right)-c_{12}+\alpha b^{1}(y)\right] \varphi(y)+c_{12} \psi(y)=\lambda \varphi(y), \\
\frac{D_{2}}{2} \psi_{y y}^{\prime \prime}+\left[\beta\left(c_{22}(0,0)+c_{21}\right)-c_{21}+\alpha b^{2}(y)\right] \psi(y)+c_{21} \varphi(y)=\lambda \psi(y), \\
y \in(-1,1), \varphi_{y}^{1}( \pm 1)=\psi_{y}^{1}( \pm 1)=0 .
\end{array}
$$

Let $\lambda(\alpha, \beta)$ be the eigenvalue having maximal real part. Such $\lambda(\alpha, \beta)$ is single and real. The wave velocity $c^{*}$ is the solution of the following equation:

$$
\inf _{z} \sup _{\alpha, \beta}\left[\alpha c^{*}+(\beta-1) z-\lambda(\alpha, \beta)\right]=0 .
$$

The parameters $\alpha, \beta, z$ vary in the real line.

REMARK 4. Suppose that $x \in R^{r}$ and let, in equations (1), the terms of the form $\left(D_{i} / 2\right)\left(\partial^{2} u / \partial x^{2}\right)$ be replaced by $\frac{1}{2} \sum_{k, l=1}^{r} a_{i}^{k l}\left(\partial^{2} u / \partial x^{k} \partial x^{l}\right)$, where $a_{i}^{k l}, i=1,2$, are constants, the matrices $\left(a_{1}^{k l}\right)$ and $\left(a_{2}^{k l}\right)$ being positive definite. The initial functions are assumed to have the form $g_{1}(x)=a \chi_{0}(x), g_{2}(x)=b \chi_{0}(x)$, where $\chi_{0}(x)$ is the indicator function of some half-space. Since the diffusion is, generally speaking, nonisotropic now, the velocity of the wave front propagation will depend on direction. Simple calculation shows that the wave propagation velocity in the direction $e=\left(e_{1}, \ldots, e_{r}\right)$ is assigned by the same formula as in $R^{1}$ (see Theorem 2), if one puts $D_{i}=\sum_{k, l=1}^{r} a_{i}^{k l} e_{k} e_{l}, i=1,2$.

REMARK 5. Theorem 2 may be slightly reformulated. We shall let $u^{\varepsilon}(t, x)=$ $u(t / \varepsilon, x / \varepsilon), v^{\varepsilon}(t, x)=v(t / \varepsilon, x / \varepsilon)$. If $u(t, x), v(t, x)$ is the solution of problem (1), then the functions $u^{\varepsilon}(t, x), v^{\varepsilon}(t, x)$ satisfy the system of equations:

$$
\begin{aligned}
& \frac{\partial u^{\varepsilon}}{\partial t}=\varepsilon \frac{D_{1}}{2} \frac{\partial^{2} u^{\varepsilon}}{\partial x^{2}}+\frac{1}{\varepsilon}\left[c_{11}\left(u^{\varepsilon}, v^{\varepsilon}\right) u^{\varepsilon}+c_{12} v^{\varepsilon}\right], \\
& \frac{\partial v^{\varepsilon}}{\partial t}=\varepsilon \frac{D_{2}}{2} \frac{\partial^{2} v^{\varepsilon}}{\partial x^{2}}+\frac{1}{\varepsilon}\left[c_{22}\left(u^{\varepsilon}, v^{\varepsilon}\right) v^{\varepsilon}+c_{21} u^{\varepsilon}\right] .
\end{aligned}
$$

Theorem 2 straightforwardly implies the following

THEOREM 2'. Suppose that the hypotheses of Theorem 2 are fulfilled. Then, no matter what the $h>0$,

$$
\lim _{\varepsilon \rightarrow 0} u^{\varepsilon}(t, x)=\lim _{\varepsilon \rightarrow 0} v^{\varepsilon}(t, x)=0 \text { for } x>c^{*} t
$$

uniformly in $\left\{(t, x): x-c^{*} t>h\right\}$;

$$
\lim _{\varepsilon \rightarrow 0} u^{\varepsilon}(t, x)=a, \quad \lim _{\varepsilon \rightarrow 0} v^{\varepsilon}(t, x)=b \quad \text { for } x<c^{*} t
$$

uniformly in $\left\{(t, x): x-c^{*} t<-h\right\}$.

In such a formulation, Theorem $2^{\prime}$ may be generalized to the case when the coefficients of system (22) depend on $x$. In the nonhomogeneous case, such effects as, for example, "appearance of new sources" may occur, analogously to what happens in the case of one equation [2]. 


\section{REFERENCES}

1. A. N. Kolmogorov, I. G. Petrovskii and N. S. Piskunov, A study of the equation of diffusion with increase in the quantity of matter, and its application to a biological problem, Bjul. Moskov. Gos. Univ. 1, 1-72.

2. M. I. Freidlin, Propagation of a concentration wave in the presence of random motion associated with the growth of a substance, Soviet Math. Dokl. 20 (1979), 503-507.

3. __ Quasi-linear parabolic equations, and measures on a function space, Funkcional. Anal. i Priložen. 1 (1967), 74-82.

4. __ Average principle and theorems on large deviations, Russian Math. Surveys 33 (1978).

5. A. D. Wentzell and M. I. Freidlin, Fluctuations in dynamical systems caused by small random pertubations, “Nauka”, Moscow, 1979.

Moscow, U.S.S.R. 